\title{
Analysis of Interpreting Process in Translation from the Perspective of Schema Theory
}

\author{
Hu Qiyun \\ Shanghai Polytechnic University \\ Email: qyhu@sspu.edu.cn
}

\begin{abstract}
As a term frequently used in psychology, psycholinguistics, cognitive science, anthropology, and artificial science, Schema (plural schemata or schemas) means "an active organization of past reactions, or of past experiences, which must always be supposed to be operating in any well-adapted organic response." (Bartlett, 1932, P.201). Given the consideration that few work has been conducted to analyze interpreting process from the perspective of schema theory, the article discusses the major kinds of schemata used in interpreting: linguistic schemata, content schemata and formal schemata and studies the effectiveness of each schemata in the interpreting process. Press Conferences of "two sessions" (of the National People's Congress (NPC) and the Chinese People's Political Consultative Conference (CPPCC)) are the main source of interpreting instances, which are considered to be closer to the real interpreting process. The article aims at elaborating the effectiveness of schema in interpreting process, and hopefully, to inspire people to utilize schema theory in interpreting spontaneously.
\end{abstract}

Keywords: Interpreting; schema; linguistic schemata; content schemata; formal schemata.

\section{Introduction}

In the process of globalization, the important role of interpreters is self-evident in building up the communication bridge between people who are separated by culture as well as by language. Accordingly, interpreting studies receive more and more attention. From the early empirical studies of interpreting to the current interdisciplinary researches on interpreting, scholars are attempting to find out more theoretical supports to explain interpreting from different angles. Drawing schema theory to do interpreting studies answers the call of this trend.

\section{Schema Theory}

\subsection{Definition and Features of Schema}

The term "schema" was first used by Swiss Psychologist Jean Piaget in1926, which is the basis of his Genetic Epistemology theory. In 1932, British psychologist Frederic Bartlett introduced the concept into psychology and education, defined the term as "an active organization of past reactions, or of past experiences, which must always be supposed to be operating in any well-adapted organic response."(Bartlett, 1932, p. 201) It is R.C. Anderson who expands the concept into schema theory: "larger and more complex units of knowledge exist that embody much of the what we know about general categories of objects, classes of events, and types of people. These knowledge structures are referred to as schema."(Anderson 1947, p. 129) A text, oral or written, carries no meaning by itself, it only points out directions for listeners or readers to retrieve meaning from their own pre-acquired knowledge; The structure of the pre-acquired knowledge or background knowledge is called schema, which is stored in people's long term memory; Schematic knowledge is composed of assumptions suggesting how the reality is arranged or organized; Activating one information-holder, or slot in the schema, the other relevant slots will be activated simultaneously, which make possible the whole schema serve as the script, frame, scenario for people to follow.

Schemata have the following characteristics: As a result of individual experiences and society progress, schema will be added with new information, which means schema grows. When people realize his/her 
existing schemata are not enough for the new information, s/he has to modify the existing schema accordingly, and when the new knowledge is discrepant from the existing schema, the existing schema will be restructured. What's more, schema is a hierarchical structure, and the simple schema can be embedded in the more complex ones.

\subsection{Main Categories of Schemata Concerned in the Thesis}

Thang Siew Ming claims that "besides reader' prior linguistic knowledge (linguistic schema) and level of proficiency in the second language, content schema and formal schema are equally important. (in reader's comprehension)" (Thang Siew Ming, 1997) which points out there are three basic schemata: linguistic schemata, which is the phonetic, lexical, syntactic and semantic knowledge of language being learned; Content schemata, that is the knowledge concerned about the content area of language being learned; and formal schemata involves the form related aspects of language being learned.

\section{$3 \quad$ Feasibility of Applying Schema Theory to Interpreting Process}

Interpreting has the characteristics of promptness and directness. The interpreter is supposed to comprehend the discourse quickly and correctly, and then interpret it immediately to ensure a smooth communication between the speaker and the listener. Therefore, interpreting is a busy and intense cognitive task.

According to an experiment conducted by Liu Jianfu (Liu Jianfu, 2001) to study the role of background knowledge in interpreting based on schema theory: The result of the experiment reveals that background knowledge is positively correlated with the effectiveness of interpretation. The effectiveness of interpretation is related to language proficiency, but language deficiency can be compensated by activating schema.

Schema theory holds that the decoding, encoding, storage and extraction of new information depend on people's existing schema. In addition, when cognition task is busy, or is distracted, the role of schemata is very influential. It can help us reasonably allocate the cognitive resources to the corresponding cognitive objectives, thereby reducing pressure of cognition, in further, improving the cognitive efficiency. To conclude, the role of schema in interpreting, which is a busy and intense cognitive task, is self-evident.

\section{Application of Schema Theory to Interpreting Process Analysis}

\subsection{Linguistic Schemata and Interpreting}

Linguistic schemata are the prerequisite to interpreting, in the following part, the functions of linguistic schemata in interpreting will be elaborated from phonological level and lexical level.

\subsubsection{Phonological schemata and interpreting}

The first level of language processing will always be phonological one: the interpreter hears a series of phonological units, based on which s/he extracts meaning.

In the each year's press conferences of the "two sessions" (of the NPC and the CPPCC), many journalists' working language, in most cases, English and Chinese, is not their mother tongue. When they speak, in most cases, they can't avoid a strong accent. The professional interpreters are supposed to analyze the acoustic features of the accents, in other words, to establish a specific phonological schema for each kind of accent, and distinguish what s/he hears when necessary.

For example, in 2011, a French journalist: "Mr. Prime minister, North Africa has been experimenting tremendous changes since the beginning of the year. And the world is now observing China which is perusing its course. Can we say that China has created its own model in the mode of politics, economics and culture? ..." All the stress falls at the end of the words, and she confused /b/and /p/, /t/and /d/. This is the phonological schema of a typical French accent.

\subsubsection{Terminological schemata and interpreting}

For a skilled interpreter, possessing the simple and basic working languages knowledge for daily communication is inadequate, words and expressions in a special domain require attention as well, known as terminology. 
As previously mentioned, "Schemata are always organized meaningfully." (Anderson, 1977, p. 418) Schema is a knowledge structure linking every element surrounding the one concept, and the whole completes the meaning of the specific schema. To build a terminological schema, we must find the relationships between terms and form a coherent meaning. The following example is the terminological schema of "income gap of China" which was mentioned in one two session's press conference.

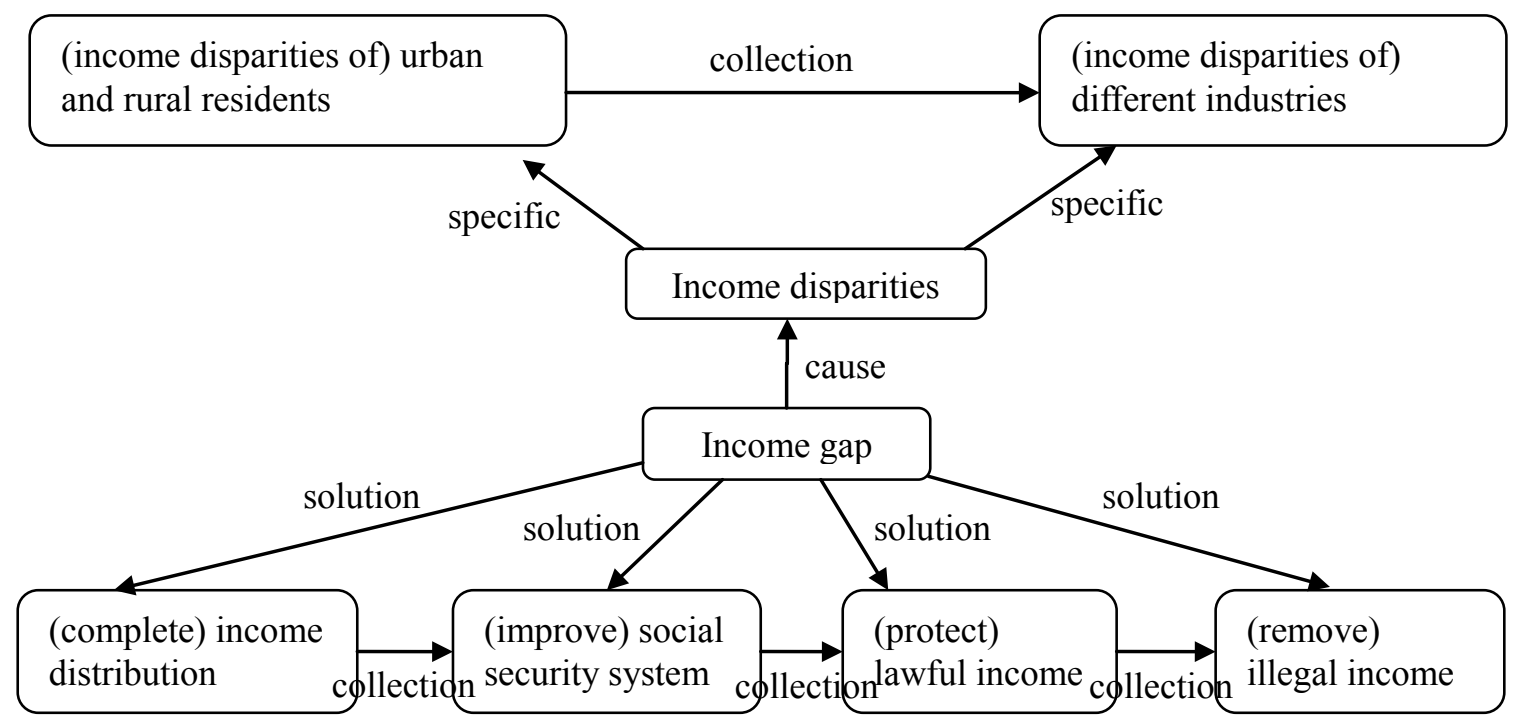

Terminological Schemata for Income Gap in China

\subsection{Content Schemata and Interpreting}

Content schemata contain the background knowledge of the content domain of a discourse and the topic being talked about. For the interpreter, it includes both bicultural background knowledge of and the familiarity with the topic.

\subsubsection{Cultural schemata and interpreting}

Cultural schemata "portray not only the world of physical objects and events, but also abstract worlds of social interaction, discourse, and even word meaning." (D'Andrade, 1995, p. 55)

The listeners may be confused with: "hear loud thunders but see few rain drops" translated from "政府 对非公经济的支持是雷声大, 雨点小。”

Now let's take a look at the interpreter's version, "it is also the view of some privately run enterprises in China that they have heard loud thunders but seen few rain drops in terms of the central government's support for the nonpublic sector or much said but little done." The content followed "in term of " transmitted not only the intention of speaker for using the idiom, but also Chinese culture.

What drives her to make the decision — - the interpreter's bicultural schemata. According to schema theory, no text is fully explicit, and the function of content schemata is to provide basis for filling gaps in the text, and finally, to form a coherent interpretation. Bicultural schemata guarantee that the interpreter will correctly perceive the meaning of source language, permit the interpreter to take into account the cultural schemata of target language listeners, in other words, what cultural symbols make sense to them, and what means nothing to them at all.

\subsubsection{Topic schemata and interpreting}

The very concept of "topic" does not only means the subject people are talking about or writing on, it also implies something or somebody receive much attention at the present time. In the government press conferences, if the interpreter is not familiar with the topic, s/he may make many pauses and hesitations, slow down the utterance, even make serious mistakes in rendition.

For example: once the premier mentioned,“最近美国码头工人协会授予我一个'美国工人最佳之友奖”'If the interpreter was not familiar with the topic here, s/he would have difficulties in translating “美国码头 工人协会” into “International Longshoreman Association" and “美国工人最佳之友奖” into “The best 
friend of American workers".

\subsection{Formal Schemata and Interpreting}

Formal schemata involve the background knowledge of genre. Genres are "conventionalized forms of texts which reflect the functions and goals involved in particular social occasions as well as the purposes of the participants in them." (Kress, 1985, p. 89 ) By saying "conventionalized", it means that the information of the text (spoken or written) is arranged in a relatively fixed form. To draw out the "conventionalize forms" of different texts types, "schema abstraction" (Anderson, 1947) will be used. Schema abstraction requires people to extract the general tendency of a set of instances, and to form a schematic description of a category from this general tendency.

We have genres for spoken text, such as lectures, ceremonial speeches, monologues in operas and so on. The two session's press conference, by schema abstraction, displays its own genre traits. Since it follows a temporal sequence, a linear structure is utilized to express the genre schemata of Premier's press conference:

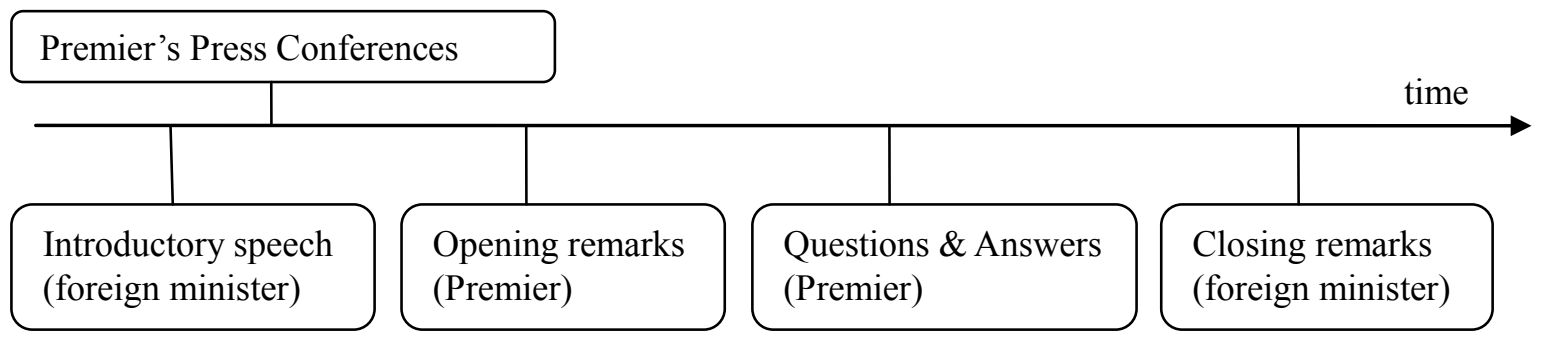

Genre Schemata of two session's press conferences

The most important aspect of Genre Schemata is that they imply the allocation of information. According to the schemata above, the interpreter is advised to focus on important information in the main body, i.e. the part of premier taking questions from the press. Whereas for the less important information--- the remarks in the beginning and at the end, most interpreters would say having no translation difficulties, but still some details require the interpreter to pay due attention.

\section{Conclusions}

The ultimate task of the interpreter is to guarantee a smooth flow of information between the speaker and the listener. To complete this task, the interpreter must constantly balance the speaker's schemata and that of the listener. This thesis classifies schemata into linguistic schemata, content schemata and formal schemata. Each schema facilitates interpreting activities at different levels:

First, with pre-acquired schemata, the interpreter can well interact with the oral texts. The higher the schema is, the better perception of the interpreter. As emphasized for so many times, besides the interpreter's language proficiency, interpreting requires the extra-linguistic knowledge of the interpreter as well. In other words, the interpreter must construct more abundant schemata.

Second, through the use of schemata, the interpreter can easily predict the allocation of information and distribute due attention to it. This is extremely helpful in listening process of interpreting, the interpreter can listen with emphasis since s/he has already born in mind in which part the important information may occur, and s/he can foresee the obstacles that may occur and draw on according techniques to deal with them. The better the interpreter is able to venture correct expectations, the fewer efforts are needed to seek for confirmation through the material, as a result the cognitive pressure is greatly reduced and the efficiency of comprehension is raised.

Third, given the fact that both the speaker and the listener have their own schemata, the interpreter can spontaneously alter expression to ensure the fluency of communication and satisfy listeners' expectations.

Fourth, schemata also propose standards on interpretation: accurate terminology, clear logic line, concise rendering on secondary information, and appropriate style of the interpreted version. 
Fifth, schemata point out directions for building schemata in interpreter training. According to schemata theory, whenever we encounter a situation, our existing schemata will be activated to process it, in return, it brings changes to our schemata. The input information is expanding the existing schemata by adding new information; or we adjust details of the existing schemata to process the new information; or our existing schemata fail to deal the input information even if we make some alterations, then our schemata need to be substituted with a new one.

The ultimate goal of schemata is to reduce cognitive tension in highly extensive interpreting activity and assists the interpreter to perform better.

\section{References}

1. Anderson, J. R. (1947). Cognitive Psychology and Its Implications. San Francisco: Freeman Press

2. Anderson R. C. (1977). The Notion of Schemata and the Educational Enterprise: General Discussion of the Conference. In Anderson R. C., Spiro R. J. and Montague W. E. (Eds.), Schooling and the Acquisition of Knowledge, (pp 415-431). New Jersey: Lawrence Erlbaum Associates.

3. Anderson R. C., Reynolds R. E., Schallert D. L., \& Goetz E. T. (1977). Frameworks for Comprehending Discourse. American Educational Research Journal, 14, 367-381.

4. Bartelett, F. C. (1932). Remembering: An Experimental and Social Psychology. Cambridge: Cambridge University Press.

5. D'Andrade R. (1995). The Development of Cognitive Anthropology. Cambridge: Cambridge University

6. Kress, G. (1985). Linguistic Progresses in Sociocultural Practice. Victoria: Deakin. University Press.

7. Thang Siew Ming. (1997). Induced Content Schema VS Induced Linguistic Schema-Which is more Beneficial for Malaysian ESL Readers? RELC Journal, 28, 107.

8. Liu Jianfu. A Schema-Theoretic View of the Role of Background Knowledge in Oral Interpretation[J]. Journal of PLA University of Foreign Languages, Vol.24 No.6 Nov. 2001, 70-74 